\title{
Ghrelin reduces cerebral ischemic injury in rats by reducing M1 microglia/macrophages
}

\author{
Rong Tian, Gengsheng Mao \\ Department of Neurosurgery, The Third Medical Centre Chinese PLA (People's Liberation Army) General Hospital, \\ Beijing, China
}

The purpose of this study was to investigate the effect of Ghrelin on the polarization of microglia/ macrophages after cerebral ischemia (CI) in rats. 60 wild-type SD rats were randomly divided into sham group, CI group, CI+Ghrelin group, 20 rats in each group. The modified Longa suture method was used to establish the middle cerebral artery occlusion (MCAO) model in rats. Before surgery, Ghrelin was injected subcutaneously (100 $\mu \mathrm{g} / \mathrm{kg}$, twice a day) for 4 consecutive weeks. After modeling, neurological function scores were performed with three behavioral experiments: mNSS score, Corner test, and Rotarod test, to evaluate the recovery of neurological function after Ghrelin treatment. At the same time, the brain tissues were collected and stained with 2,3,5triphenyltetrazolium chloride (TTC) to detect the cerebral infarct volume. RT-qPCR was used to detect the expression of TNF- $\alpha$ and IL-1 $\beta$ in the ischemic brain tissue, and the TUNEL staining was used to detect the apoptosis of brain tissue. Flow cytometry was used to detect the percentage of M1 type microglia/macrophages which were isolated by trypsin digestion of fresh cerebral cortex. Then, the Western blotting and immunofluorescence method were used to detect the phosphorylation level of AKT (P-AKT) and AKT. Compared with the CI group, the neurological function of the rats in the CI+Ghrelin group was dramatically improved, and the cerebral infarction area was dramatically reduced. At the same time, the expression of TNF- $\alpha$ and IL- $1 \beta$ in the ischemic brain tissue of rats in the CI+Ghrelin group decreased, and the apoptotic cells in the brain tissue also decreased. Compared with the CI treatment group, the activation of M1 microglia/macrophages in the cortex of the ischemic side of the infarct and the peri-infarct area in the CI+Ghrelin group was dramatically inhibited. At the same time, the ratio of P-AKT/AKT of the brain tissue in the CI+Ghrelin group was dramatically higher than that of the CI group. In the rat cerebral ischemia model, Ghrelin can promote the repair of brain damage and the recovery of neurological function after ischemia. Its mechanism may be related to activating AKT to selectively reduce M1 microglia/macrophages, reducing inflammation and cell apoptosis in brain tissue.

Key words: Cerebral ischemia; ghrelin; microglia/macrophage.

Correspondence: Gengsheng Mao, MD. Department of Neurosurgery, The Third Medical Centre Chinese PLA (People's Liberation Army) General Hospital, 69 Yongding Road, Haidian District, Beijing 100039, China. E-mail: kety@longupp.com

Contributions: All the authors made a substantive intellectual contribution, read and approved the final version of the manuscript and agreed to be accountable for all aspects of the work.

Conflict of interest: The authors declare that they have no competing interests, and all authors confirm accuracy.

Ethics approval: This study was approved by the Animal Ethics Committee of the Animal Center of Third Medical Centre Chinese PLA General Hospital (16-PL-EA-3421).

Availability of data and materials: The data used to support the findings of this study are available from the corresponding author on reasonable request. 


\section{Introduction}

Stroke has become the second leading cause of death in the world and the number one cause of death in China. ${ }^{1}$ Among them, ischemic stroke is the main type of stroke. Ischemic stroke can cause permanent necrosis and apoptosis of nerve cells, which in turn leads to impaired neurological function in patients. Cerebral ischemia can trigger a series of cascade reactions characterized by inflammation. Thrombolytic drugs can treat cerebral ischemia, but there is a strict time window. ${ }^{2}$ Commonly used antiplatelet aggregation drugs, such as aspirin, can be used to treat cerebral ischemia, but studies have reported that long-term combined use of antiplatelet aggregation drugs can increase the risk of cerebral hemorrhage. Therefore, more and more attention has been paid to the research on alternative medicines for the treatment of cerebral infarction/ischemia.

Microglia are a type of innate immune cells that exist in the central nervous system. They are very sensitive to changes in the extracellular environment. When brain tissue is subject to noxious stimuli such as infection, ${ }^{3}$ ischemia, ${ }^{4}$ and trauma, ${ }^{5}$ microglia will change from a resting state (M0) to an active state. The rest state (M0) is transformed into an activated state, and the activated microglia are divided into a classical activation state (M1 type) and a selective activation state (M2 type). When cerebral ischemia occurs, due to the destruction of the blood-brain barrier, the mononuclear-macrophages in the blood will leak, infiltrate, and recruit to the injured area, and they participate in the inflammatory response together with activated microglia. ${ }^{5}$ Therefore, we collectively call them microglia-macrophages. M1 can produce interleukin (IL)-1, IL-6, IL-23, tumor necrosis factor $\alpha$ (TNF- $\alpha$ ) and other pro-inflammatory factors, as well as CCL2, CXCL9 and CXCL10 and other chemokines to promote inflammation and tissue damage, causing toxic effects on neurons. ${ }^{6} \mathrm{M} 2$ can secrete anti-inflammatory factors such as IL-4, IL-10 and transforming growth factor $\beta$, inhibit excessive inflammatory response, promote tissue repair and neuron regeneration, and promote vascular regeneration and tissue repair. ${ }^{7}$ The environment of the infarct zone is conducive to the differentiation of macrophages into M1 type, further aggravating brain tissue damage. Inhibiting the differentiation of macrophages into the M1 phenotype has a protective effect on the mouse model of cerebral infarction. ${ }^{8}$ Therefore, inhibiting the differentiation of microglia/macrophages to the M1 phenotype and reducing the inflammatory response may be a new treatment strategy for cerebral infarction. Regulating the phenotypic differentiation of microglia/macrophages has been considered as a new research direction for brain nerve repair.

Ghrelin is an endogenous active peptide composed of 28 amino acids with a relative molecular mass of 33,000 . As an endogenous ligand of growth hormone secretagogue, it can dramatically stimulate the release of growth hormone secretagogue. ${ }^{9}$ Most of the Ghrelin in the human body is mainly secreted by $\alpha$ cells in the gastric mucosa, and is widely distributed in the pancreas, heart, hypothalamus, cerebral cortex, brainstem and other organs. Studies have shown that Ghrelin can not only promote the release of growth hormone secretagogue hormone, but also can regulate food intake, gastrointestinal motility, sleep cycle and other aspects. ${ }^{10}$ In recent years, some scholars have found that the receptors of Ghrelin are densely distributed in the hippocampus, and the administration of exogenous Ghrelin can dramatically increase the density of dendritic spines in the CA1 area of the hippocampus, and achieve the effect of improving learning and memory. ${ }^{11}$ In AD, studies have shown that the expression level of Ghrelin has changed dramatically, and the use of Ghrelin or Ghrelin receptor agonists can dramatically improve AD-related pathological phe- nomena. ${ }^{12,13}$ This may be that Ghrelin promotes its growth by activating the proteasome in nerve cells, so as to play a protective role of neurons. However, whether the neuroprotective effect of Ghrelin is related to the activation of microglia/macrophages has not been reported in the literature.

This project established a rat model of focal cerebral ischemia to observe the scores of neurological deficits, as well as the expression of TNF- $\alpha$, IL-1 $\beta$, cell apoptosis and the percentage of M1type microglia/macrophages, to further explore the potential mechanism of the neuroprotective effect of Ghrelin on cerebral ischemia injury, so as to provide theoretical basis and laboratory basis for its clinical application.

\section{Material and Methods}

\section{Experimental animal}

Healthy male Sprague-Dawley rats (8-10 weeks old, body weight 280-320 g) were purchased from the Animal Center of Third Medical Centre Chinese PLA General Hospital. Feeding conditions: temperature $22 \pm 2^{\circ} \mathrm{C}$, humidity $50 \pm 10 \%, 12 \mathrm{~h} / 12 \mathrm{~h}$ light cycle. Animals have free access to food and water. The experiment process followed the "Guiding Opinions on the Good Treatment of Laboratory Animals" issued by the Ministry of Science and Technology of the People's Republic of China. This study was approved by the Animal Ethics Committee of the Animal Center of Third Medical Centre Chinese PLA General Hospital (16-PL-EA-3421).

\section{Animal grouping and administration}

After 60 rats were adaptively fed for 2 weeks, all rats were numbered and divided into 3 groups according to the random number table: sham operation group, cerebral ischemia (CI) group, $\mathrm{CI}+$ Ghrelin group, 20 rats in each group. The specific drugs of each group were given as follows: The CI+Ghrelin group was given Ghrelin subcutaneous injection $(100 \mu \mathrm{g} / \mathrm{kg}$, twice a day; Tianpu Biochemical Pharmaceutical, Guangzhou, China) for 4 consecutive weeks. ${ }^{14}$ The Sham group and the CI group were injected with the same volume of normal saline.

\section{Cerebral ischemia model}

The middle cerebral artery occlusion (MCAO) model was established by referring to the improved Longa suture method. Operation steps: i) The rats were weighed and fasted for $12 \mathrm{~h}$ before operation; ii) the rats were anesthetized by intraperitoneal injection of $2 \%$ pentobarbital sodium; iii) after the anesthesia was successful, the rats were fixed on the operating table in the supine position, skin was fully prepared for disinfection, the skin was cut along the middle of the neck, the subcutaneous tissue and each artery was bluntly separated with a glass minute needle. The proximal end of the common carotid artery and the distal end of the external carotid artery were ligated, the distal end of the internal carotid artery was clamped by the arterial vascular clip, and the distal end of the common carotid artery was reserved for use. Then a small opening in the common carotid artery was cut with ophthalmic scissors and inserted the MCAO thread plug. When the thread plug entered the internal carotid artery, the vascular clip was removed and inserted it slowly. When the distance from the bifurcation of the common carotid artery was $18 \pm 0.5 \mathrm{~mm}$, the backup line fixation line plug was tightened. Finally, the skin incision was sutured and disinfected. After the operation, the rats were placed on a constant temperature electric blanket at $37^{\circ} \mathrm{C}$ to keep warm. 


\section{Rat neurological behavioral score}

With reference to the method of the previous study, all experimental rats were given a basic behavioral score before MCAO surgery, and animals with abnormal scores were eliminated. Rats in the CI group and $\mathrm{CI}+\mathrm{Ghrelin}$ group received Modified Neurological Severity Score (mNSS) test and Corner test behavioral tests every other day after $24 \mathrm{~h}$ of surgery, and scored to 30 days. Before the operation and 1, 7, 14, 28 days after the operation, the Rotarod test was used to detect the recovery of animal nerve function (after setting the system parameters, put the rats on the device and start the rotation mode, the system will automatically save the data). The experiments were conducted in a double-blind manner, and the results of behavioral experiments were observed and recorded at the same time.

\section{Measurement of cerebral infarct volume}

The volume of cerebral infarction was measured by 2,3,5triphenyltetrazolium chloride (TTC) staining method. 7 days after CI, 5 rats in each group were randomly selected and brain tissues were taken after anesthesia, and immediately placed horizontally in the refrigerator at $-20^{\circ} \mathrm{C}$ for about $20 \mathrm{~min}$, and then cut into 5 slices along the coronal position, with a thickness of about $2 \mathrm{~mm}$. The slices were placed in $1 \%$ TTC buffer (Life Technology, Wuhan, China), incubated at $37^{\circ} \mathrm{C}$ in the dark for $30 \mathrm{~min}$, and then placed in $4 \%$ paraformaldehyde for fixation for $24 \mathrm{~h}$. Image Pro Plus 6 software was used to determine the percentage of cerebral infarction volume.

\section{Preparation of frozen tissue sections}

On the $7^{\text {th }}$ day after ischemia, the rats were anesthetized by intraperitoneal injection of $2 \%$ pentobarbital sodium, and the ascending aorta was intubated through the left ventricle. The rats were quickly perfused with normal saline for $2 \mathrm{~min}$, and then perfused with $4 \%$ paraformaldehyde. After the perfusion, the rat skull was removed, and the brain tissue was carefully taken out. After fixing the brain tissue in $4 \%$ paraformaldehyde for $12 \mathrm{~h}$, it was immersed in $15 \%$ sucrose solution and $30 \%$ sucrose solution for cryo-protection. After quick freezing, continuous coronal sectioning was performed on a cryostat, the thickness of the brain slice was $15-20 \mu \mathrm{m}$, and the slices were stored in a refrigerator at $-80^{\circ} \mathrm{C}$.

\section{TUNEL staining}

After fixation and rinsing, the sections were incubated at $37^{\circ} \mathrm{C}$ with a blocking solution containing $10 \%$ goat serum, $3 \%$ BSA and $0.3 \%$ TritonX-100 for $1 \mathrm{~h}$ to block the non-specific binding sites. Then the sections were stained according to the instructions of the TUNEL kit (Jian Cheng, Nanjing, China), and the positive spots were observed under a fluorescence microscope after DAPI staining of the nucleus. The apoptotic index was assessed by TUNEL positive cells in all cells in each microscope fields $(n=3)$.

\section{Immunofluorescent staining}

After the above treatment, the frozen sections were incubated with anti-AKT monoclonal antibody (1:1000, Abcam, Cambridge, MA, USA) and microglia marker (Iba 1, 1:500, Abcam) overnight at $4{ }^{\circ} \mathrm{C}$. On the second day, PBS was used to rinse three times, and fluorescent secondary antibodys (goat anti-rabbit-FITC, 1:100, Sigma Aldrich, St. Louis, MO, USA and goat anti-mouse-Cy3, 1:200, Thermo Fisher Scientific, Waltham, MA, USA) diluted with PBS were added dropwise to incubate for $1 \mathrm{~h}$ at room temperature. After three rinses with PBS, the fluorescent mounting solution was used for mounting. Next, they were observed under a fluorescence microscope and photographed. The co-expression immunopositivity index was assessed by the ratio of $\mathrm{p}$-AKT positive cells/Iba-1 positive cells.

\section{Flow cytometry}

After the rats were anesthetized, the cerebral cortex of the ischemic side was taken. The brain tissue was cut into pieces in a $60 \mathrm{~mm}$ cell culture dish, $0.25 \%$ trypsin was added, and digested at $37^{\circ} \mathrm{C}$ for $20 \mathrm{~min}$. The serum was used to stop the digestion. After centrifugation at $1,000 \mathrm{rpm}$ for $5 \mathrm{~min}$, the 1640 medium was used to resuspend the pellet that was filtered through a 200 -mesh screen. $2 \mathrm{~mL}$ erythrocyte lysate was added to it, bathed in $37^{\circ} \mathrm{C}$ water for 2-3 min, centrifuged and washed twice with PBS. CD45, CD11b and isotype control antibodies (Thermo Fisher Scientific) were added respectively and then mixed, and incubated at room temperature for $30 \mathrm{~min}$. After washing with PBS once, PBS was used to resuspend the cells and tested on the flow cytometer. All flowcytometry studies were performed using a BD Biosciences FACSCanto II or BD FACS LSR Fortessa. All data were analysed using FlowJo 10.07 (Tree Star).

\section{RNA extraction and RT-qPCR}

Seven days after the CI model, 5 rats in each group were randomly selected. After the rats were sacrificed by cervical dislocation, about $100 \mathrm{mg}$ of brain tissue from the ischemic injury area was collected. After low temperature homogenization, the total RNA was extracted by the TRIzol method and reversed according to Takara Bio Inc. (Shiga, Japan). Recorded the steps of the kit instructions, reverse transcribed the cDNA on a PCR machine, and then added the reaction system according to the group, $10 \mu \mathrm{L}$ per well, 3 replicate wells. The reaction conditions are: pre-denaturation at $94^{\circ} \mathrm{C}$ for $4 \mathrm{~min}$, denaturation at $94^{\circ} \mathrm{C}$ for $30 \mathrm{~s}$, annealing at $58^{\circ} \mathrm{C}$ for $30 \mathrm{~s}$, extension at $72^{\circ} \mathrm{C}$ for $45 \mathrm{~s}$, a total of 40 cycles, and a final extension at $72^{\circ} \mathrm{C}$ for $5 \mathrm{~min}$. The $2^{-\Delta \Delta \mathrm{Ct}}$ method was used to calculate the relative expression of the above genes. Primers are shown in Table 1.

\section{Western blotting}

Eighty mg of the ischemic area and surrounding brain tissue of each group of rats were taken, then $100 \mu \mathrm{L}$ of RIPA lysis solution containing PMSF (Kaiji, Nanjing, China) was added, and ultrasonically lyse for $15 \mathrm{~min}$ on ice. After centrifugation at $4^{\circ} \mathrm{C}, 12,000$ $\mathrm{r} / \mathrm{min}$ for $20 \mathrm{~min}$, the supernatant was transferred to another centrifuge tube, and the protein was quantified by Bradford method. Thirty $\mu \mathrm{g}$ total protein was separated by sodium dodecyl sulfatepolyacrylamide gel electrophoresis, and then the protein was transferred to nitrocellulose (NC; Thermo Fisher Scientific) membrane. After 5\% skimmed milk powder was used for blocking for $2 \mathrm{~h}$, the primary antibodies (p-AKT, AKT, Abcam, Mouse, 1:2000; GAPDH, Abcam, 1:5000) were added at $4^{\circ} \mathrm{C}$ overnight. The next

Table 1. Real time PCR primers.

\begin{tabular}{lll} 
Gene name & Forward $\left(5^{\prime}>3^{\prime}\right)$ & Reverse $\left(5^{\prime}>3^{\prime}\right)$ \\
$T N F-\alpha$ & CCTCTCTCTAATCAGCCCTCTG & GAGGACCTGGGAGTAGATGAG \\
$I L-1 \beta$ & GCAACTGTTCCTGACTCAACT & ATCTTTTGGGGTCCGTCAACT \\
\hline GAPDH & ACAACTTTGGTATCGTGGAAGG & GCCATCACGCCACAGTTTC \\
\hline
\end{tabular}


day, HRP-labeled secondary antibodies were added and incubated at room temperature for $2 \mathrm{~h}$. After TBST rinses for 3 times, the enhanced chemiluminescence (ECL; Camilo, Nanjing, China) was added to the $\mathrm{NC}$ membrane for color development according to the requirements of the substrate kit. Image $\mathrm{J}$ software was used for grayscale analysis.

\section{Statistics analysis}

All statistical analysis was processed by SPSS20.0 statistical software, and the data were expressed as mean \pm standard deviation. Student's $t$-test was used for comparison between the two groups. One or two-way ANOVA was used for data analysis among multiple groups. The statistical analysis of behavioral data adopts repeated measure ANOVA. A p-value $<0.05$ is considered significant.

\section{Results}

Ghrelin dramatically improves neurobehavioral function and reduces cerebral infarction volume after cerebral ischemic injury

In order to research the effect of Ghrelin on the neurological function recovery of rats after $\mathrm{CI}$, neurological function scores were conducted with three behavioral experiments including mNSS score, Corner test and Rota-rod test before surgery and within 30 days after ischemia to evaluate the neurological function recovery after administration. As shown in Figure 1, there was no neurobehavioral function injury in the sham operation group. After ischemia, behavioral scores increased in both CI group and $\mathrm{CI}+$ Ghrelin group, indicating that the rats after MCAO have obvious neuromotor dysfunction, such as left forelimb unable to stretch, signs of rotation to the left, standing instability, falling to the ischemic side or turning to the ischemic side during tail lift. Ghrelin can dramatically improve the neurological symptoms of rats after 10 days of administration (Figure 1A). In the Corner test experiment, Ghrelin dramatically reduced the number of times the rats turned to the right side paralyzed (Figure 1B). In the Rota-rod test experiment, compared with the CI group, the rats in the Ghrelin administration group stayed in the rotating rod dramatically longer, and with time, the gap gradually increased (Figure 1C). The above experimental results suggest that Ghrelin treatment promotes the recovery of neurological function after CI. At the same time, we assessed the degree of CI injury by TTC staining. Compared with the sham group, the cerebral infarction volume of the CI group and the CI+Ghrelin group increased dramatically, and compared with the CI group, the cerebral infarction volume of the $\mathrm{CI}+$ Ghrelin group decreased dramatically (Figure 1D). In summary, Ghrelin can dramatically relieve the neurobehavioral function and reduce the volume of cerebral infarction after CI injury.
A

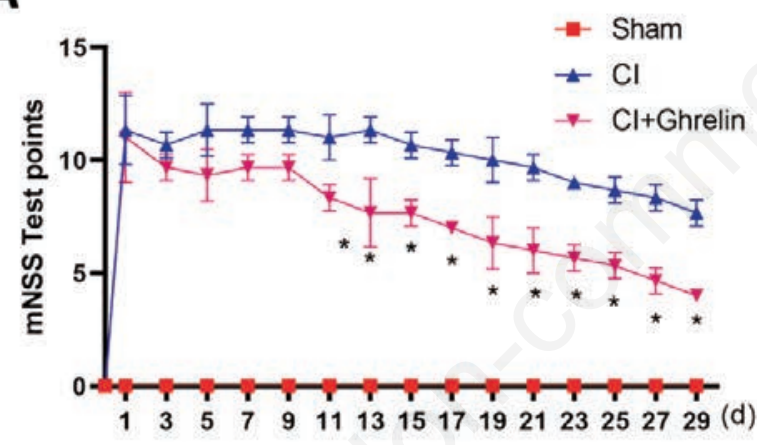

C

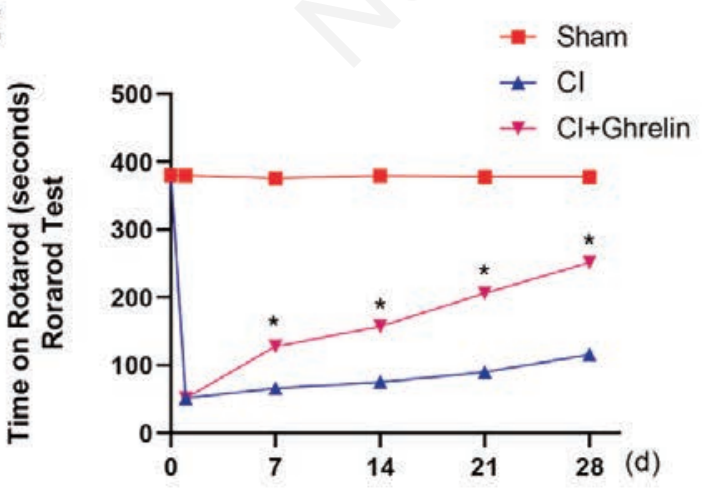

B

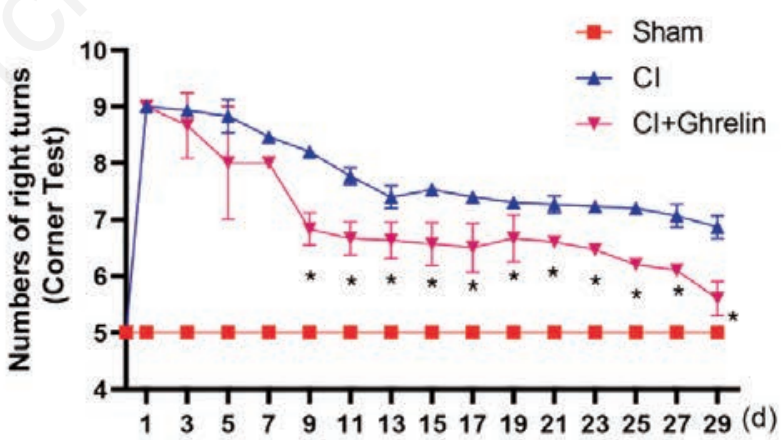

D

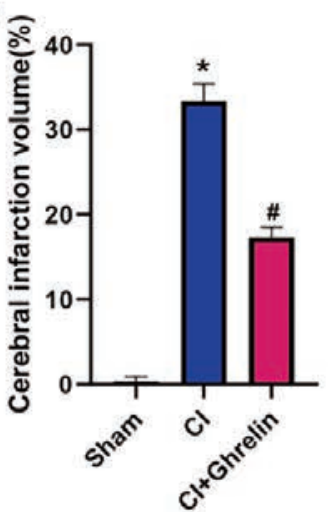

Figure 1. Ghrelin dramatically improves neurobehavioral function and reduces cerebral infarction volume after cerebral ischemic injury. A) Modified Neurological Severity Scores ( $\mathrm{mNSS}$ ) ( $\mathrm{n}=17$ for sham group, $\mathrm{n}=16$ for CI group, $\mathrm{n}=16$ for CI+Ghrelin group). B) Corner test ( $\mathrm{n}=18$ for sham group, $\mathrm{n}=17$ for CI group, $\mathrm{n}=16$ for $\mathrm{CI}+\mathrm{Ghrelin}$ group). C) Bota-Bod test ( $\mathrm{n}=16$ per group). D) TTC staining was used to detect the infarct volume of brain tissue ( $n=5$ per group). ${ }^{*} p<0.05,{ }^{*} p<0.05$. 
Ghrelin inhibits inflammation and apoptosis in the ischemic area of rats with cerebral ischemia

In order to explore the effect of Ghrelin on the expression of inflammatory factor mRNA in rats with CI injury, this study implemented RT-qPCR detection of related inflammatory factors. The results of the study showed that the expression of inflammatory factors (IL-1 $\beta$, TNF- $\alpha$ ) in the cerebral ischemic side of CI rats was dramatically upregulated; compared with CI group, the expression of inflammatory factors in the $\mathrm{CI}+\mathrm{Ghrelin}$ group was reduced (Figure 2 A,B). At the same time, TUNEL staining showed that compared with the sham group, cell apoptosis in the CI group was dramatically increased, and compared with the CI group, the cell apoptosis in the $\mathrm{CI}+$ Ghrelin group was dramatically reduced (Figure 2C). Therefore, the above results indicated that Ghrelin can inhibit inflammation and apoptosis in the ischemic area of cerebral ischemic rats.

\section{Ghrelin promotes the phosphorylation of AKT in the} brain after ischemia

To further explore the mechanism of Ghrelin. We use Western Blot to detect the phosphorylation level of AKT in brain tissue. The results showed that compared with the sham group, the AKT phosphorylation level in the CI group was increased. Compared with the CI group, the AKT phosphorylation level of the CI+Ghrelin group was dramatically higher (Figure 3A). Studies have shown that AKT can be expressed in microglia in in vivo and in vitro experiments. ${ }^{15}$ After ischemia, immunofluorescence staining was used to label the microglia marker (Iba 1) and P-AKT (Figure 3B). The results showed that the brain tissue can express Iba 1 and PAKT, and Ghrelin can increase the expression of P-AKT. The above results proved that the AKT pathway in the cerebral ischemic penumbra of the striatum can be activated in microglia.

\section{Ghrelin reduces the percentage of M1 type microglia/macrophages}

In order to study the effect of Ghrelin on inducing microglia polarization in the brain after ischemia. After the rats were treated with CI model, 5 rats were randomly selected from the sham group, CI group and $\mathrm{CI}+\mathrm{Ghrelin}$ group respectively. After execution, the tissue in the ischemic penumbra of bilateral striatum was quickly separated. Compared with the sham group, the percentage of M1 type microglia/macrophages in the CI group increased dra-
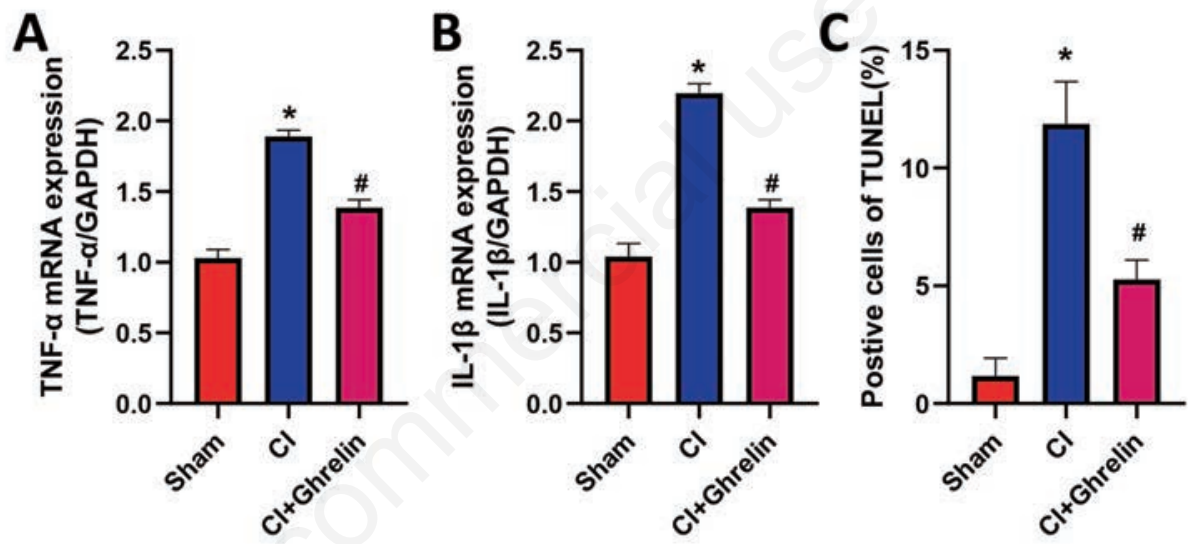

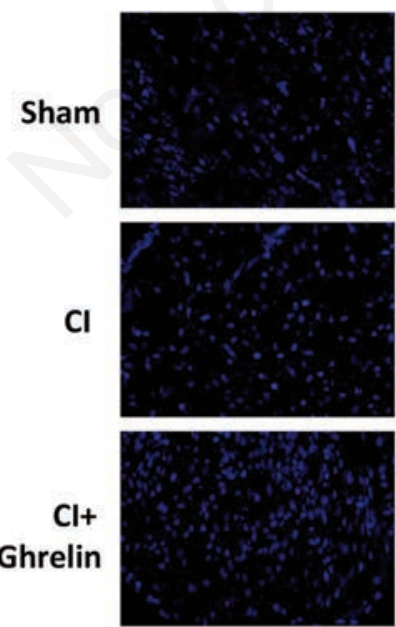

DAPI
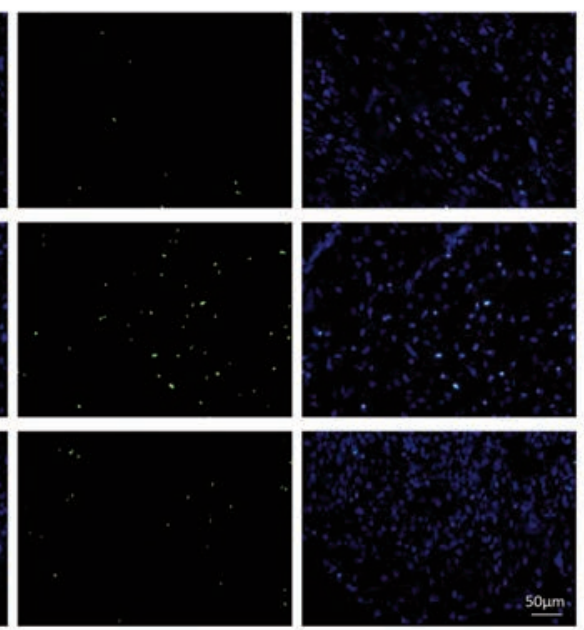

TUNEL

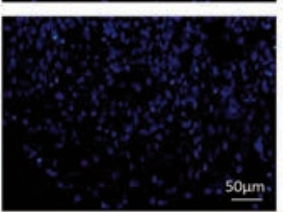

MERGE

Figure 2. Ghrelin inhibits inflammation and apoptosis in the ischemic area of rats with cerebral ischemia. A) RT-qPCR was used to detect the mRNA of TNF- $\alpha$ in brain tissue, GAPDH was used as an internal reference. B) RT-qPCR was used to detect the mRNA of IL-1 $\beta$ in brain tissue, GAPDH was used as an internal reference. C) TUNEL staining was used to detect the apoptosis cell of brain tissue; the apoptotic index was assessed by TUNEL positive cells in all cells in each microscope fields $(\mathrm{n}=3) .{ }^{*} \mathrm{p}<0.05,{ }^{\#} \mathrm{p}<0.05$. 
A

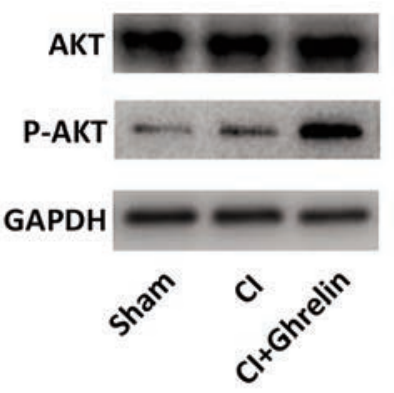

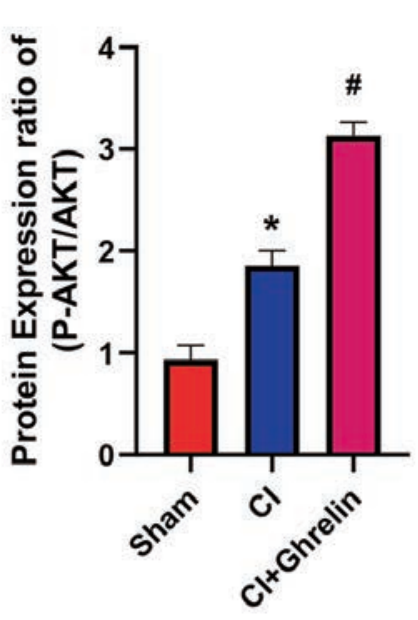

B

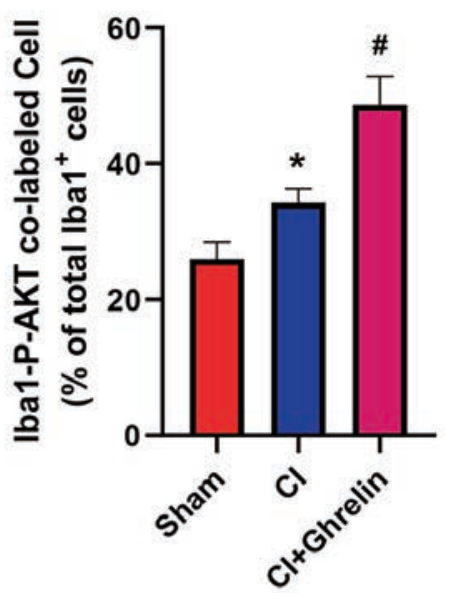

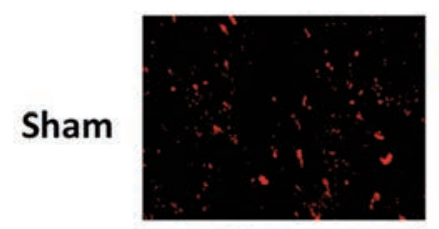
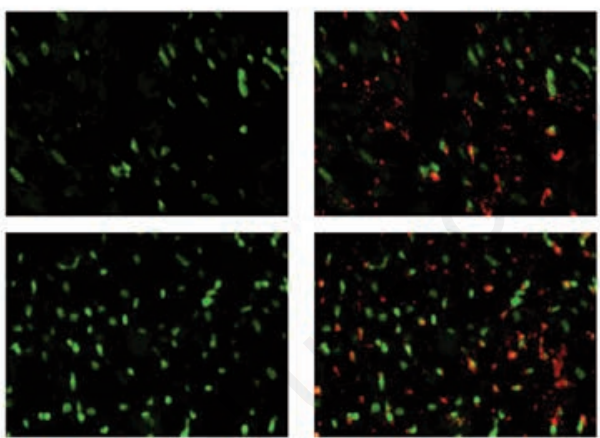

$\mathrm{Cl}+$

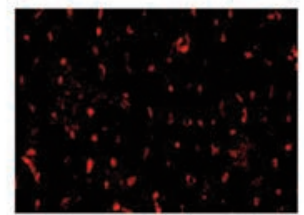

Iba 1

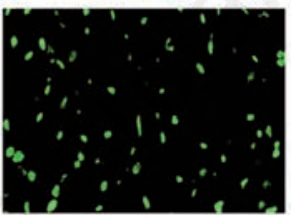

P-AKT

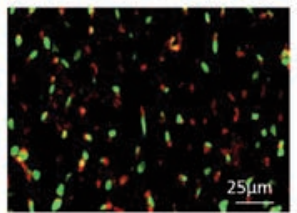

MERGE

Figure 3. Ghrelin promotes the phosphorylation of AKT in the brain after ischemia. A) Western blotting was used to detect the protein of P-AKT, AKT in brain tissue, and quantitative analysis of $\mathrm{p}$-Akt /AKT ratio; ${ }^{*} \mathrm{p}<\mathbf{0 . 0 5},{ }^{\#} \mathrm{p}<\mathbf{0 . 0 5}$. B) Immunofluorescent staining was used to detect the colocalization of P-AKT (green) with the microglial marker Iba 1 (red).

A

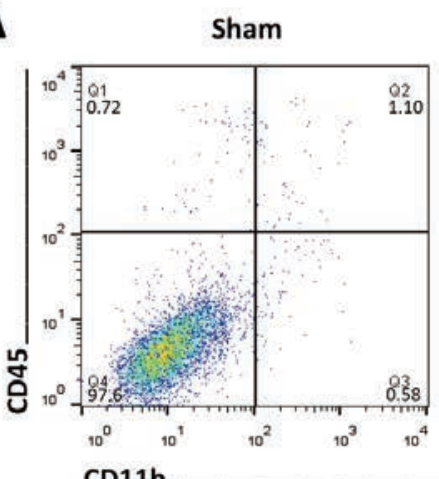

Cl

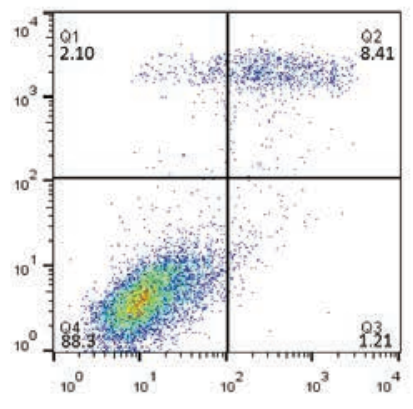

$\mathrm{Cl}+$ Ghrelin

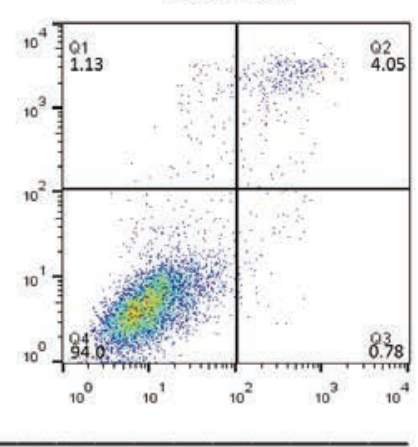

B

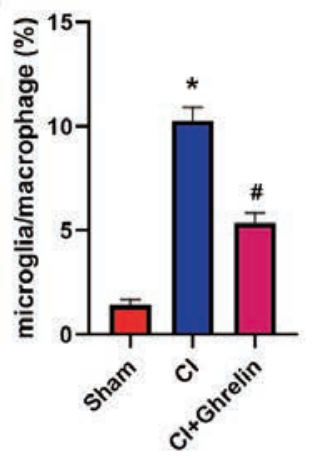

Figure 4. Ghrelin reduces the percentage of M1 type microglia/macrophages. A) Flow cytometry pictures of rat CI microglia/macrophages in each group. B) The percentage of $\mathrm{Cl}$ microglia/macrophages of rats in each group. ${ }^{*} \mathrm{p}<0.05,{ }^{\#} \mathrm{p}<0.05$. 
matically. Compared with the CI group, the percentage of M1 type microglia/macrophages in the $\mathrm{CI}+\mathrm{Ghrelin}$ group decreased dramatically (Figure 4 A,B). The above results indicated that Ghrelin can reduce the percentage of M1 type microglia/macrophages.

\section{Discussion}

In this study, we found that in the rat model of focal CI, Ghrelin can reduce M1 microglia/macrophages in the ischemic penumbra zone by activating the AKT pathway, and also promote the recovery of nerve function after ischemia.

After ischemic stroke, the different polarization states of microglia change dynamically with the course of the disease. Studies have shown that in the early stage of ischemia, M2 type microglia rapidly increase within $24 \mathrm{~h}$ of injury, and their phenotypic markers are highly expressed in the core area of ischemia. ${ }^{16}$ At this time, microglia can reduce tissue damage, enhance nerve regeneration and tissue repair, and exert neuroprotective effects. Subsequently, the M2 type gradually transformed to the M1 type. The M1 type microglia markers began to increase on the 3rd day after ischemia, and became dominant on the $7^{\text {th }}$ day, and continued for several weeks. ${ }^{17}$ At this time, activated microglia extends from the ischemic core area to the peripheral area of the infarction- the ischemic penumbra area. The function of M1 type microglia is mainly to promote the release of inflammatory factors, which is not conducive to the regeneration and repair of the late stage of CI, but aggravates brain damage. Therefore, we can inhibit the transition from M2 to M1 and induce the transition from M1 to M2 to increase the ratio of $\mathrm{M} 2 / \mathrm{M} 1$, thereby reducing neuroinflammation after CI. This experiment verifies that Ghrelin has a significant therapeutic effect on rats with CI injury in vivo, which is reflected in that Ghrelin reduces the cerebral infarct area on the ischemic side of CI rats and promotes the improvement of behavior.

In this study, the inflammatory cytokines IL- $1 \beta$ and TNF- $\alpha$ were selected as the research objects to explore the effect of Ghrelin on the mRNA expression of inflammatory factors in rats with CI injury. TNF- $\alpha$ is synthesized by macrophages, and its overexpression can lead to the activation of neutrophils and lymphocytes and the expression of adhesion factors, which in turn aggravates ischemic brain tissue damage. ${ }^{18,19} \mathrm{IL}-1 \beta$ is mainly derived from glial cells, neurons and vascular endothelial cells, and its overexpression causes a large number of neutrophils to enter the central nervous system, release harmful inflammatory mediators, and aggravate brain damage..$^{20,21}$ And we confirmed that Ghrelin can inhibit the expression of IL- $1 \beta$ and TNF- $\alpha$, thereby inhibiting the inflammatory response in the ischemic area.

Ghrelin promotes the phosphorylation of AKT in the brain after ischemia. The AKT pathway can inhibit inflammation through activation of the mTOR complex. Activated Akt phosphorylates multiple downstream targets that also regulate apoptosis related proteins including members of the Bcl-2 protein family, $\mathrm{BAD}$, and so on. Ghrelin negatively regulates apoptotic and inflammatory responses by AKT pathway.

In this study, we demonstrated that Ghrelin can promote brain injury repair and neurological function recovery after brain ischemia, and the mechanism may be related to the activation of AKT pathway, thereby reducing M1 microglia at the ischemic site, it will provide a new repair mechanism and a new drug target for the repair of ischemic brain injury.

\section{References}

1. Wu S, Wu B, Liu M, Chen Z, Wang W, Anderson CS et al. Stroke in China: advances and challenges in epidemiology, prevention, and management. Lancet Neurol 2019;18:394-405.

2. Wardlaw JM, Murray V, Berge E, Del ZG. Thrombolysis for acute ischaemic stroke. Cochrane Database Syst Rev 2014;(7):D213.

3. Garber C, Soung A, Vollmer LL, Kanmogne M, Last A, Brown $\mathrm{J}$, et al. T cells promote microglia-mediated synaptic elimination and cognitive dysfunction during recovery from neuropathogenic flaviviruses. Nat Neurosci 2019;22:1276-88.

4. Cengiz P, Zafer D, Chandrashekhar JH, Chanana V, Bogost J, Waldman A, et al. Developmental differences in microglia morphology and gene expression during normal brain development and in response to hypoxia-ischemia. Neurochem Int 2019;127:137-47.

5. Tsuda M. Microglia-mediated regulation of neuropathic pain: Molecular and cellular mechanisms. Biol Pharm Bull 2019;42:1959-68.

6. Przanowski P, Mondal SS, Cabaj A, Debski KJ, Wojtas B, Gielniewski B, et al. Open chromatin landscape of rat microglia upon proinvasive or inflammatory polarization. Glia 2019;67:2312-28.

7. Cherry JD, Olschowka JA, O'Banion MK. Neuroinflammation and M2 microglia: the good, the bad, and the inflamed. J Neuroinflammation 2014;11:98.

8. Tang Z, Gan Y, Liu Q, Yin JX, Liu Q, Shi J, et al. CX3CR1 deficiency suppresses activation and neurotoxicity of microglia/macrophage in experimental ischemic stroke. J Neuroinflammation 2014;11:26.

9. Kang S, Moon NR, Kim DS, Kim SH, Park S. Central acylated ghrelin improves memory function and hippocampal AMPK activation and partly reverses the impairment of energy and glucose metabolism in rats infused with beta-amyloid. Peptides 2015;71:84-93.

10. Zeng M, Huang C, Zheng H, Chen Q, He W, Deng Y. Effects of ghrelin on iNOS-derived NO promoted LPS-induced pulmonary alveolar epithelial A549 cells apoptosis. Cell Physiol Biochem 2018;49:1840-55.

11. Diano S, Farr SA, Benoit SC, McNay EC, Da SI, Horvath B, et al. Ghrelin controls hippocampal spine synapse density and memory performance. Nat Neurosci 2006;9:381-8.

12. Jeon SG, Hong SB, Nam Y, Tae J, Yoo A, Song EJ, et al. Ghrelin in Alzheimer's disease: Pathologic roles and therapeutic implications. Ageing Res Rev 2019;55:100945.

13. Theodoropoulou A, Metallinos IC, Psyrogiannis A, Vagenakis GA, Kyriazopoulou V. Ghrelin and leptin secretion in patients with moderate Alzheimer's disease. J Nutr Health Aging 2012; 16:472-7.

14. Zhou F, Xu J, Ying GY, Wang L, Zhu XD. Ghrelin attenuated cerebral ischemia reperfusion injury in rats. CNS Neurosci Ther 2012;18:945-946.

15. Chen J, Wang Z, Zheng Z, Chen Y, Khor S, Shi K, et al. Neuron and microglia/macrophage-derived FGF10 activate neuronal FGFR2/PI3K/Akt signaling and inhibit microglia/macrophages TLR4/NF-kappaB-dependent neuroinflammation to improve functional recovery after spinal cord injury. Cell Death Dis 2017;8:e3090.

16. Hu X, Li P, Guo Y, Wang H, Leak RK, Chen S, et al. Microglia/macrophage polarization dynamics reveal novel mechanism of injury expansion after focal cerebral ischemia. Stroke 2012;43:3063-70.

17. Benakis C, Garcia-Bonilla L, Iadecola C, Anrather J. The role of microglia and myeloid immune cells in acute cerebral 
ischemia. Front Cell Neurosci 2014;8:461.

18. Zarruk JG, Greenhalgh AD, David S. Microglia and macrophages differ in their inflammatory profile after permanent brain ischemia. Exp Neurol 2018;301:120-32.

19. Warrington JP, Drummond HA, Granger JP, Ryan MJ. Placental ischemia-induced increases in brain water content and cerebrovascular permeability: role of TNF-alpha. Am J
Physiol Regul Integr Comp Physiol 2015;309:R1425-31.

20. Esenwa CC, Elkind MS. Inflammatory risk factors, biomarkers and associated therapy in ischaemic stroke. Nat Rev Neurol 2016;12:594-604.

21. Zhao SC, Ma LS, Chu ZH, Xu H, Wu WQ, Liu F. Regulation of microglial activation in stroke. Acta Pharmacol Sin 2017;38:445-58.

Received for publication: 21 October 2021. Accepted for publication: 18 December 2021.

This work is licensed under a Creative Commons Attribution-NonCommercial 4.0 International License (CC BY-NC 4.0).

(C) Copyright: the Author(s), 2022

Licensee PAGEPress, Italy

European Journal of Histochemistry 2022; 66:3350

doi:10.4081/ejh.2022.3350 